OPEN A novel crosstalk between BRCA 1 and

SUBJECT AREAS:

OVARIAN CANCER

CANCER METABOLISM

Received

11 July 2014

Accepted

1 October 2014

Published

17 October 2014

Correspondence and requests for materials should be addressed to

D.L. (leeda@ymail. com) or Q.Y. (yangq@ sj-hospital.org)

\section{sirtuin 1 in ovarian cancer}

\author{
Da Li ${ }^{1}$, Fang-Fang Bi ${ }^{1}$, Na-Na Chen ${ }^{2}$, Ji-Min $\mathrm{Cao}^{3}$, Wu-Ping Sun ${ }^{4}$, Yi-Ming Zhou ${ }^{4}$, Chun-Yan Li ${ }^{5}$ \\ \& Qing Yang'
}

\begin{abstract}
'Department of Obstetrics and Gynecology, Shengjing Hospital of China Medical University, Shenyang 1 10004, China,
${ }^{2}$ Department of Molecular Immunology, Graduate School of Medicine, Nagoya University, Nagoya 466-8550, Japan,

${ }^{3}$ Department of Physiology and Pathophysiology, Institute of Basic Medical Sciences, Chinese Academy of Medical Sciences, School of Basic Medicine Peking Union Medical College, Beijing 100005, China, ${ }^{4}$ Division of Cell Signaling, National Institute for Physiological Sciences, Okazaki 444-8787, Japan, ${ }^{5}$ Department of Histology and Embryology, Institute of Basic Medical Sciences, Chinese Academy of Medical Sciences, School of Basic Medicine Peking Union Medical College, Beijing 100005, China.
\end{abstract}

BRCA mutations are the main known hereditary factors for ovarian cancer. Notably, emerging evidence has led to considerable interest in the role of sirtuin 1 (SIRT1) in ovarian cancer development. However, dynamic crosstalk between BRCA1 and SIRT1 is poorly understood. Here, we showed that: (i) BRCA1 inactivation events (mutation, promoter methylation, or knockdown) were accompanied by decreased SIRT1 levels and increased nicotinamide adenine dinucleotide (NAD) levels and a subsequent increase in SIRT1 activity; (ii) overexpression of BRCA1 resulted in increased SIRT1 levels, an impairment in NAD synthesis, and a subsequent inhibition of SIRT1 activity; and (iii) intracellular NAD levels were largely responsible for regulating SIRT1 activity, and BRCA1 expression patterns correlated with SIRT1 levels and NAD levels correlated with SIRT1 activity in human ovarian cancer specimens. Interestingly, although BRCA1 inactivation events inhibited SIRT1 expression, they led to a substantial increase in NAD levels that enhanced NAD-related SIRT1 activity. This is a special BRCA1-mediated compensatory mechanism for the maintenance of SIRT1 function. Therefore, these results highlight a novel interaction between BRCA1 and SIRT1, which may be beneficial for the dynamic balance between BRCA1-related biologic processes and SIRT1-related energy metabolism and stress response.

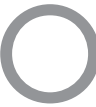

varian cancer is the most lethal gynecologic malignancy in women worldwide ${ }^{1}$. To date, although the exact cause of ovarian cancer remains largely unknown, BRCA mutations are the main known hereditary factors for the initiation and progression of ovarian cancer $^{2}$. BRCA1 is a tumor suppressor gene involved in multiple cellular processes ${ }^{3}$. Recent research has confirmed that BRCA1 is an important transcriptional regulator, and that BRCA1 depletion leads to changes in approximately $7 \%$ of the mRNAs expressed in cancer cells $^{3}$. Moreover, our previous study indicated that there are wide ranges of transcriptional regulation, epigenetic patterns and metabolic differences between BRCA1 dysfunction and the basal phenotype ${ }^{4-9}$. Sirtuin 1 (SIRT1), a nicotinamide adenine dinucleotide (NAD)-dependent histone deacetylase that is intrinsically linked to metabolism, stress responses, epigenetic regulation and genome stability ${ }^{10,11}$. Notably, emerging evidence has suggested a possible link between BRCA1 and SIRT1. For example, BRCA1 is an important modulator of numerous cellular stresses such as metabolic stress ${ }^{12,13}$, oxidative stress ${ }^{14}$, and replication stress ${ }^{15}$. Similarly, SIRT1 is a key metabolic sensor in response to cellular stress and is responsible for the changes in metabolic homeostasis ${ }^{16}$. In addition, it is well known that NAD is a potent activator and substrate of SIRT1, and BRCA1 has recently been proposed as a NAD-dependent metabolic switch in ovarian cancer ${ }^{17}$. However, little is known about the direct link(s) between BRCA1 and SIRT1 in ovarian cancer. For this reason, the present study was undertaken to investigate the crosstalk between BRCA1 and SIRT1 status, and to provide novel insights into the regulatory mechanism of BRCA1 and SIRT1-related ovarian cancer progression.

\section{Results}

BRCA1, rather than BRCA2, may be involved in SIRT1 expression and NAD-mediated SIRT1 activity. Intracellular NAD levels were increased in non-BRCA1-mutated and BRCA1-mutated ovarian cancers compared with their adjacent normal tissue. However, it is interesting to note that BRCA1-mutated ovarian cancer showed dramatically increased intracellular levels of NAD compared with the other three groups (Fig. 1a). Although intracellular SIRT1 levels were increased in non-BRCA1-mutated ovarian cancer compared with 

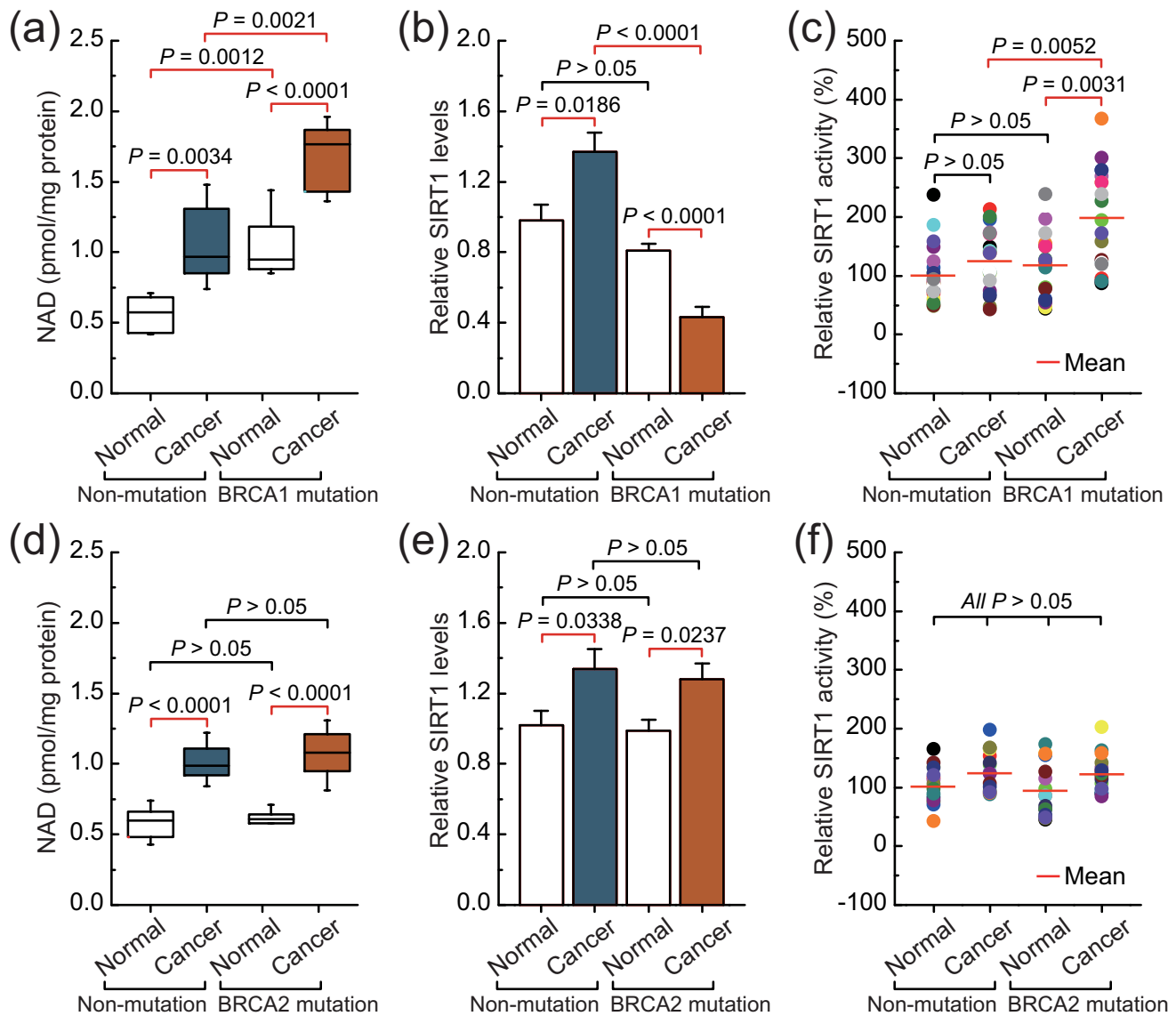

Figure $1 \mid$ Intracellular NAD levels and SIRT1 levels and activity in non-mutated, BRCA1-mutated, and BRCA2-mutated ovarian cancer. (a-c), NAD levels, and SIRT1 levels and activity were measured in 20 pairs of non-BRCA1-mutated and BRCA1-mutated ovarian cancer and their adjacent normal tissue. Bar graphs show mean \pm SD. (d-f), NAD levels, and SIRT1 levels and activity were measured in 16 pairs of non-BRCA2-mutated and BRCA2mutated ovarian cancer and their adjacent normal tissue. Bar graphs show mean \pm SD.

adjacent normal tissue, BRCA1-mutated ovarian cancer showed dramatically decreased intracellular levels of SIRT1 compared with adjacent normal tissue (Fig. 1b). Intracellular SIRT1 activity was increased in BRCA1-mutated ovarian cancer compared with adjacent normal tissue, but there was no significant difference in SIRT1 activity between non-BRCA1-mutated ovarian cancer and the adjacent normal tissue (Fig. 1c). In addition, although intracellular NAD levels and SIRT1 levels were also increased in non-BRCA2-mutated and BRCA2-mutated ovarian cancers compared with their adjacent normal tissue, there was no significant difference between the non-BRCA2-mutated and BRCA2-mutated groups, including ovarian cancer and normal ovarian tissues (Figs. 1d and e). It should be noted that there was no significant difference in SIRT1 activity among the four groups (Fig. 1f). Therefore, these results suggest that BRCA1 inactivation may be involved in the inhibition of SIRT1 expression and elevated SIRT1 activity.

BRCA1 inactivity mediated by promoter hypermethylation is accompanied by suppression of SIRT1 expression and increased SIRT1 activity. In mammals, promoter methylation is an epigenetic modification involved in regulating gene expression ${ }^{6,8,9}$. Consistent with this idea, we showed that ovarian cancer tissue with a hypermethylated BRCA1 promoter (Fig. 2a and b) displayed reduced expression of BRCA1 (Fig. 2c) compared with adjacent normal tissue. Based on these considerations, the low levels of BRCA1 mediated by promoter hypermethylation were an appropriate model for investigating the physiologic relationship between BRCA1 status and SIRT1 expression or activity. Notably, there was a marked increase in NAD levels (Fig. 2d) accompanied by enhanced SIRT1 activity (Fig. 2f) and a significant decrease in SIRT1 levels (Fig. 2e), along with a hypermethylated promoter-mediated BRCA1 deficiency. In addition, we analyzed the correlation between BRCA1 levels or NAD levels and SIRT1 levels or SIRT1 activity in 34 ovarian cancer samples (Figs. $2 \mathrm{~g}-\mathrm{j}$ ). It is interesting to note that a significant positive correlation was only observed between levels of BRCA1 and SIRT1 $(R=0.545, P<0.001$; Fig. $2 \mathrm{~g})$ and between NAD levels and SIRT1 activity $(R=0.666, P<0.001$; Fig. $2 j)$. These results indicate that BRCA1 may be responsible for the regulation of SIRT1 expression and NAD-related SIRT1 activity.

BRCA1 can regulate intracellular SIRT1 levels and NAD-related SIRT1 activity in ovarian cancer cells. To confirm the role of BRCA1 in the regulation of SIRT1 expression and activity, the effects of knockdown or overexpression of BRCA1 were evaluated in three ovarian cancer cell lines (A2780, SKOV-3, and CAOV-3) and primary ovarian cancer cells with identified BRCA1 mutations or no BRCA1 mutations. The results indicated that: (i) overexpression of BRCA1 could effectively reduce the NAD levels in A2780 and primary non-BRCA1-mutated and BRCA1-mutated ovarian cancer cells; (ii) knockdown of BRCA1 was an effective way to induce an increase of NAD levels in A2780 and primary nonBRCA1-mutated ovarian cancer cells, but NAD levels were not sensitive to BRCA1 knockdown in primary BRCA1-mutated ovarian cancer cells; and (iii) there were no significant changes in NAD levels after the knockdown or overexpression of BRCA1 in SKOV-3 and CAOV-3 cells (Fig. 3a). Interestingly, the changes in intracellular NAD levels were consistent with the tendency of SIRT1 


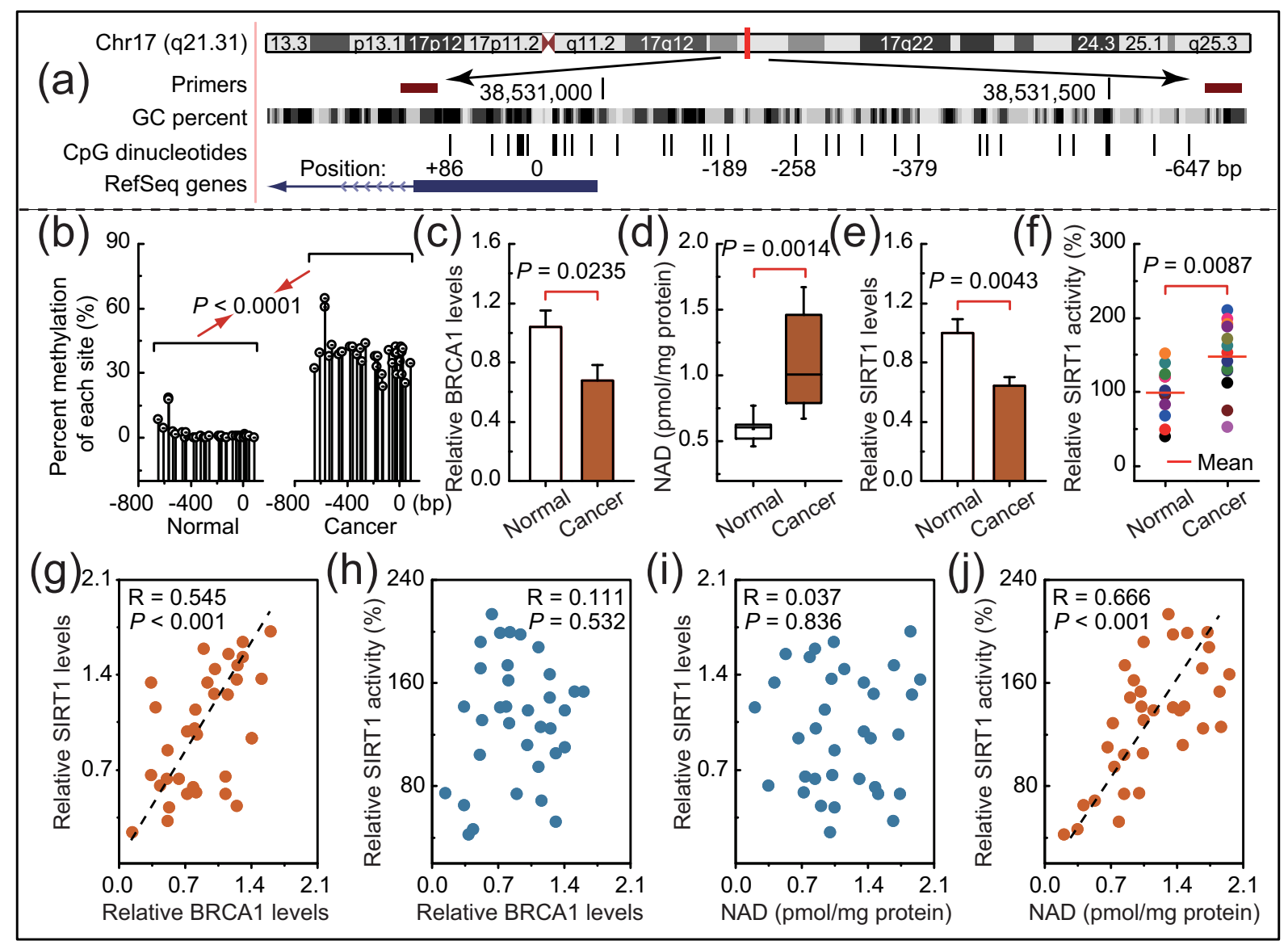

Figure 2 Intracellular NAD levels and SIRT1 levels and activity in ovarian cancer with hypermethylated promoter-mediated BRCA1 inactivation. (a), The location of CpG sites in the core promoter region of BRCA1. Genomic coordinates are shown, along with the primer-amplified fragments, GC percentage, location of individual CpG dinucleotides (dashes), and BRCA1 RefSeq gene (exon 1 is shown as a blue box and the intron is shown as an arrowed line). The arrow indicates the direction of transcription. (b), Summary of the methylation patterns of the BRCA1 promoter in Fig. 2a. The y-axis shows the mean number of methylation sites. (c-f), BRCA1 levels, NAD levels, and SIRT1 levels and activity were measured in ovarian cancer with an identified hypermethylated BRCA1 promoter and compared with those of adjacent normal tissue (unmethylated BRCA1 promoter). Bar graphs show mean \pm SD (each group, $\mathrm{n}=13$ ). ( $\mathrm{g}$ and $\mathrm{h}$ ), Correlation between BRCA1 levels and SIRT1 levels or SIRT1 activity in ovarian cancer tissues (each group, $\mathrm{n}$ $=34)$. ( $\mathrm{i}$ and $\mathrm{j}$ ), Correlation between NAD levels and SIRT1 levels or SIRT1 activity in ovarian cancer tissues (Each group, $\mathrm{n}=34$ ).

activity (Fig. 3d) rather than the intracellular SIRT1 levels (Fig. 3b and c). In addition, knockdown or overexpression of BRCA1 was an effective way to inhibit or induce SIRT1 expression, respectively (Fig. $3 b$ and c), but SIRT1 levels were not sensitive to BRCA1 knockdown in primary BRCA1-mutated ovarian cancer cells.

Intracellular NAD can induce SIRT1 activity but not SIRT1 expression in ovarian cancer cells. To further confirm the role of intracellular NAD levels in the regulation of SIRT1 activity, the effects of incubation with different concentrations of NAD and knockdown or overexpression of nicotinamide phosphoribosyltransferase (Nampt) were evaluated in ovarian cancer cell line A2780 and primary ovarian cancer cells with identified BRCA1 mutations or no BRCA1 mutations, due to these cells were sensitive to changes in NAD levels mediated by BRCA1 (as shown in Fig. 3a). SIRT1 activity was increased (Fig. 4b), accompanied by increased levels of intracellular NAD (Fig. 4a). However, intracellular SIRT1 levels were not sensitive to incubation with extracellular NAD in ovarian cancer cells (Fig. $4 \mathrm{c}$ and d). It is well known that Nampt is a rate-limiting enzyme in regenerating NAD in mammals. The results indicate that knockdown or overexpression of Nampt could effectively reduce or increase NAD levels in ovarian cancer cells (Fig. 4e). Also, reduced or increased NAD levels mediated by Nampt could effectively inhibit or induce SIRT1 activity (Fig. 4f), but not influence SIRT1 levels in ovarian cancer cells (Fig. 4g and h). These results suggest that BRCA1-mediated NAD synthesis may be responsible for the induction of SIRT1 activity.

Intracellular NAD can feedback activate BRCA1 expression in ovarian cancer cells. As already known, SIRT1 overactivation results in NAD depletion, and our previous study suggested that NAD may be a positive regulator for BRCA1 transcription in ovarian cancer cells ${ }^{17}$. Therefore, it can be speculated that SIRT1 overactivation-mediated NAD consumption may inhibit BRCA1 function. To confirm the role of SIRT1-related NAD levels in the regulation of BRCA1 levels, the effects of knockdown or overexpression of SIRT1 and incubation with different concentrations of NAD were evaluated in ovarian cancer cell line A2780 and primary ovarian cancer cells with identified BRCA1 mutations or no BRCA1 mutations, due to these cells were sensitive to changes in NAD levels mediated by BRCA1 (as shown in Fig. 3a). Notably, we observed that BRCA1 expression was upregulated, along with increased levels of intracellular NAD (Fig. 5a). In addition, increased or reduced NAD levels (Fig. 5b) mediated by the knockdown or overexpression of SIRT1 could effectively induce or inhibit BRCA1 expression in A2780 and primary non-BRCA1mutated ovarian cancer cells (Fig. 5c). 
(a)

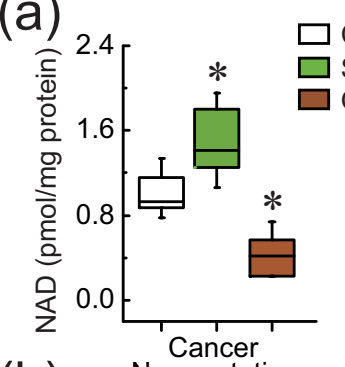

(b)
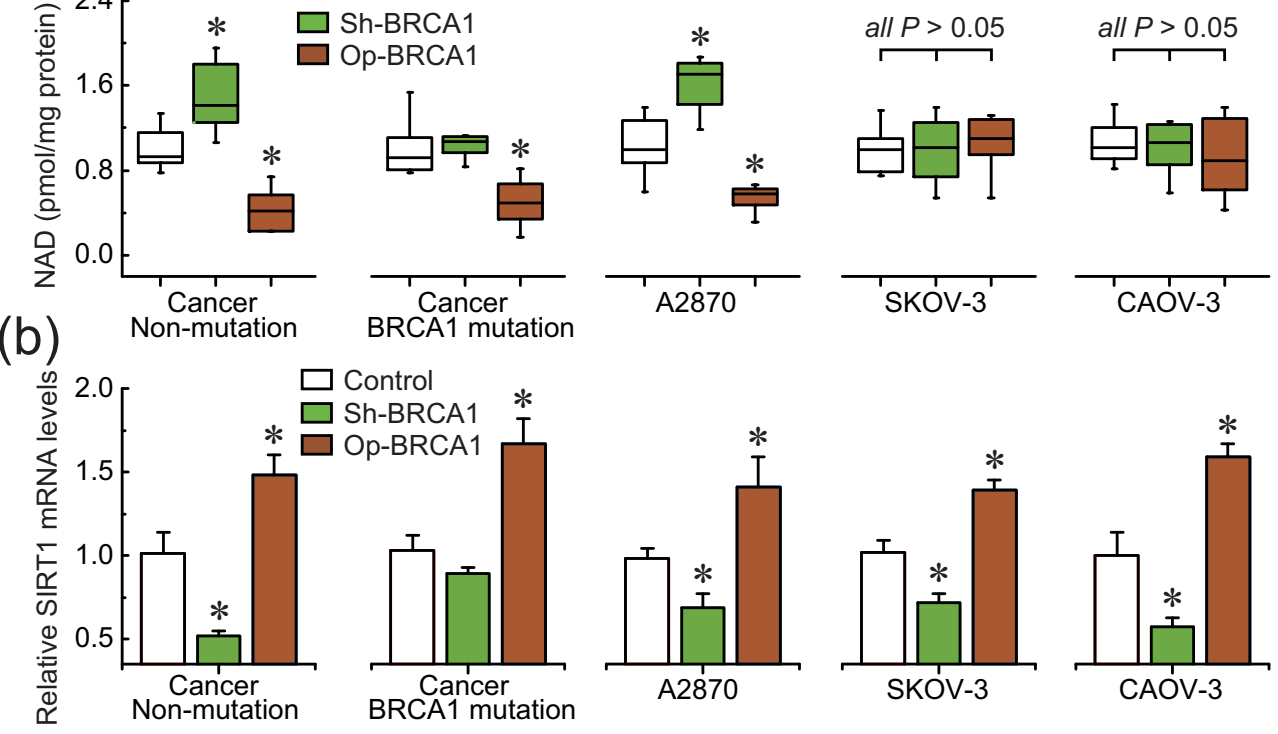

(C)
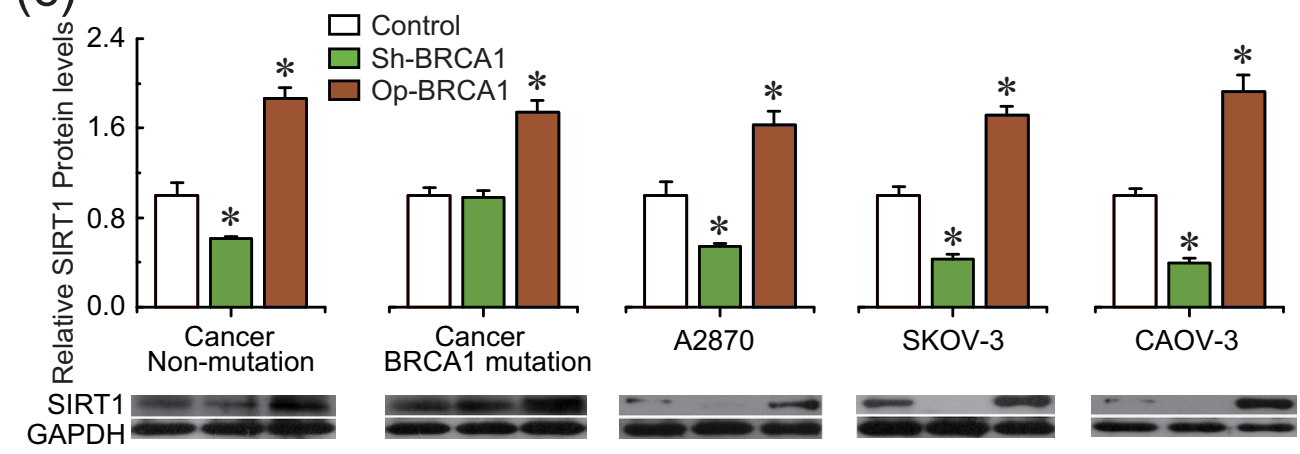

(d)
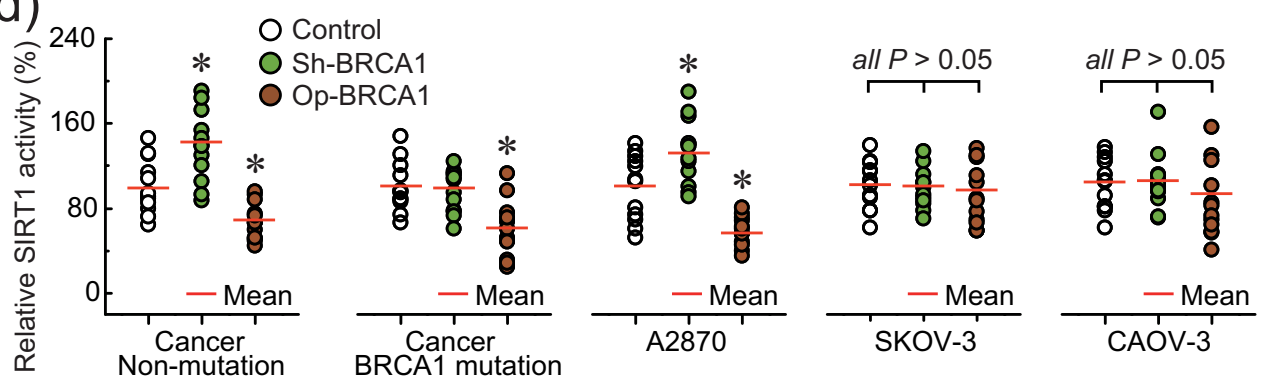

Figure 3 Effects of BRCA1 on intracellular NAD levels and SIRT1 levels and activity. (a-d), NAD levels, SIRT1 mRNA and protein levels, and SIRT1 activity after knockdown or overexpression of BRCA1 in A2780, SKOV-3, and CAOV-3 ovarian cancer cell lines (repeated 12 times), and primary nonBRCA1-mutated and BRCA1-mutated ovarian cancer cells $(\mathrm{n}=12)$. Bar graphs show mean \pm SD. Sh, shRNAs; Op, overexpression. * $P<0.05$ s. control.

\section{Discussion}

In this study, we report for the first time that BRCA1 is a positive regulator of SIRT1 levels and a negative regulator of NAD-related SIRT1 activity. Interestingly, the work presented here provides direct evidence that a BRCA1-mediated switch between SIRT1 expression and activity may have significant physiological roles in the maintenance of SIRT1-related biological processes in vivo (Fig. 6). However, NAD levels and SIRT1 expression and activity were not affected by BRCA1 knockdown in primary BRCA1-mutated ovarian cancer cells. It can be speculated that a lack of function of BRCA1 knockdown-mediated NAD or SIRT1 regulation, which may be due to BRCA1 itself, has no function along with BRCA1-mutation. In addition, as shown in Fig. 4, intracellular NAD levels may be responsible for the induction of SIRT1 activity, but we observed that nonBRCA1-mutated ovarian cancer tissues harbored higher levels of
NAD (Fig. 1a), but not higher levels of SIRT1 activity (Fig. 1c). The reason for this disparity is currently unknown but appear to comprise more complex regulatory mechanisms in ovarian cancer.

Notably, these observations further correlate the physiological properties of BRCA1 with SIRT1-related metabolism in ovarian cancer cells. For example, (i) BRCA1 inactivation not only increases reactive oxygen species generation, but also activates protective autophagy ${ }^{13,18}$. SIRT1 can positively regulate autophagy under oxidative stress ${ }^{19}$. In this regard, SIRT1 expression and imbalance in its activity may be involved in BRCA1-related oxidative stress and autophagy; (ii) Endoplasmic reticulum (ER) stress signaling plays an important part in the induction of BRCA1 expression ${ }^{20}$. However, ER stress can be attenuated by a SIRT1-dependent mechanism $^{21,22}$. Therefore, BRCA1-related elevated levels of SIRT1 may resist the adverse effects of ER stress; (iii) Both NAD and SIRT1 play 

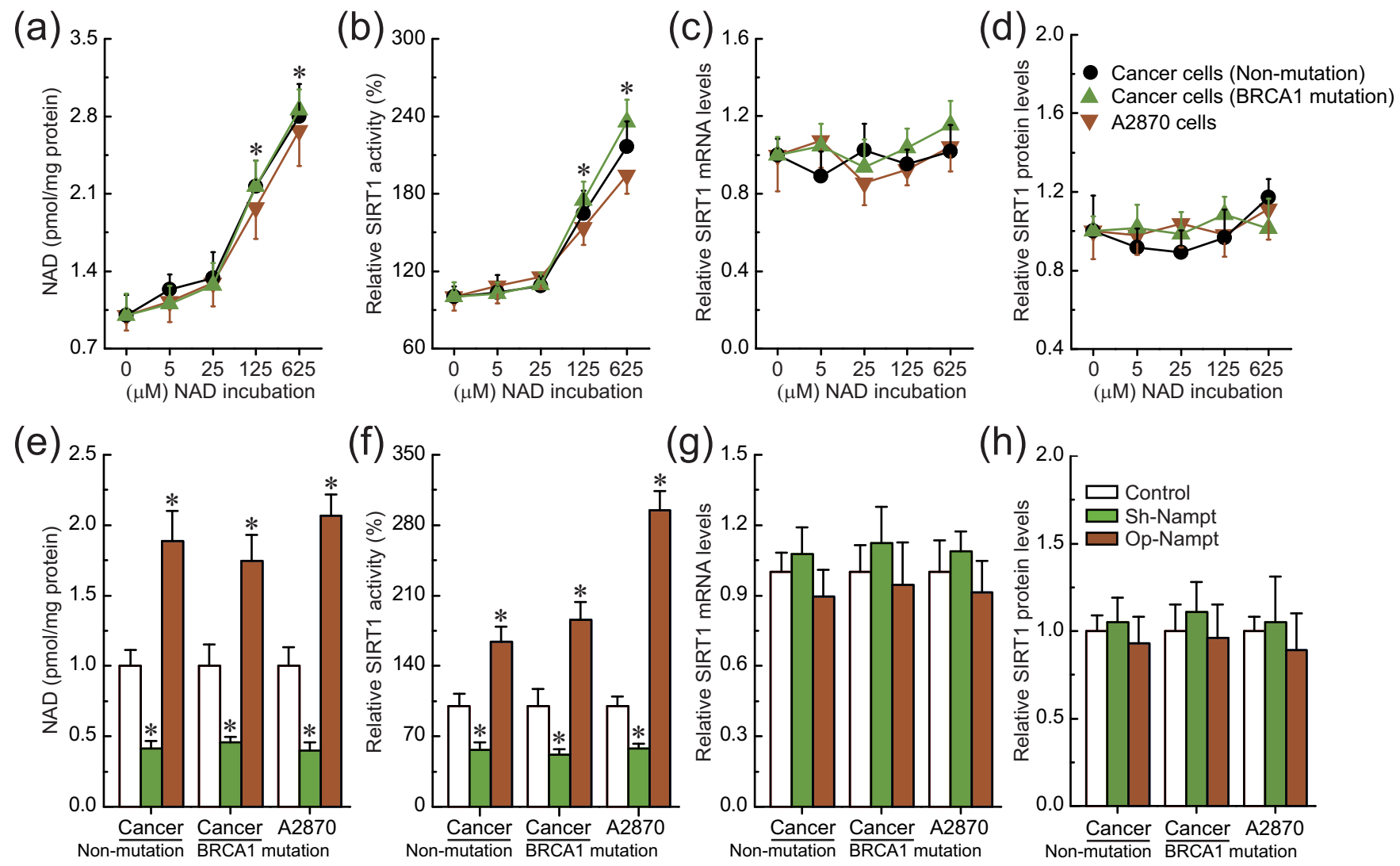

Figure 4 Effects of intracellular NAD on SIRT1 expression and activity. (a-d), Relative NAD levels, SIRT1 activity, and SIRT1 mRNA and protein levels after incubation with different concentrations of NAD in A2780 cells (repeated 12 times) and primary non-BRCA1-mutated and BRCA1-mutated ovarian cancer cells $(\mathrm{n}=12)$. 1-5: incubation with $0,5,25,125$, or $625 \mu \mathrm{M}$ NAD. Bar graphs show mean $\pm \mathrm{SD}$. ${ }^{*} P<0.05 v$ s. control. (e-h), Relative NAD levels, SIRT1 activity, and SIRT1 mRNA and protein levels after knockdown or overexpression of Nampt in A2780 cells (repeated 12 times) and primary non-BRCA1-mutated and BRCA1-mutated ovarian cancer cells $(\mathrm{n}=12)$. Bar graphs show mean $\pm \mathrm{SD}$. Sh, shRNAs; Op, overexpression. ${ }^{*} P<0.05$ vs. control.

pivotal roles in cell proliferation ${ }^{23,24}$, a substantial body of evidence indicates that loss of function of the tumor suppressor gene BRCA1 plays an important role in promoting cancer cell proliferation and survival $^{25,26}$. One can speculate that effects of BRCA1 on cell proliferation, which may involve in SIRT1 due to BRCA1 as a metabolic switch for NAD and SIRT ${ }^{17}$. However, to date, it is not fully understood how the direct or indirect interaction between BRCA1 and SIRT1 at the molecular level. But some insight was gained by a previous study that BRCA1 serves as a positive regulator of SIRT1 by binding to the promoter region ${ }^{27,28}$. These results together with our previous finding provided two novel pathways of BRCA1-NADSIRT1 activity and SIRT1-NAD-BRCA1 function (Fig. 6) ${ }^{17}$. The crosstalk may be beneficial for the dynamic balance between BRCA1-related biologic processes such as cell cycle progression, apoptosis, DNA damage repair, transcription regulation, and SIRT1-related energy metabolism and stress responses. All of this may improve our understanding of the basic molecular mechanism underlying BRCA1- and SIRT1-related ovarian cancer progression.

\section{Methods}

Ethical Statements. The research was carried out in accordance with the Declaration of Helsinki (2013) of the World Medical Association and approved by the Institutional Review Board at China Medical University.

Patients and tissue collection. Serous ovarian cancer patients were enrolled between 2010 and 2012, and all patients gave informed consent. Fresh tumor samples, adjacent normal ovarian tissues, ascites, and blood samples were obtained at the time of primary surgery before any chemotherapy or radiotherapy (20 pairs of BRCA1mutated or not, 16 pairs of BRCA2-mutated or not, and 13 pairs with hypermethylated BRCA1 promoter or not). Hematoxylin and eosin staining of the samples for histopathologic diagnosis and grading were performed by three staff pathologists using World Health Organization criteria. All patients were screened for BRCA1 and BRCA2 mutations by multiplex polymerase chain reaction (PCR). Their characteristics are given in Supplementary Table S1 and Table S2.

Cell culture and lentiviral transfection. Primary ovarian cancer cells were obtained from the ascites of patients undergoing surgery for ovarian cancer and cultured in RPMI 1640 with $10 \%$ fetal bovine serum (Invitrogen, CA, USA) as previously reported $^{4-6}$. Human A2780, SKOV-3, and CAOV-3 ovarian cancer cells were maintained in Dulbecco's Modified Eagles Medium (DMEM) with 10\% fetal bovine serum (Invitrogen). Lentiviral vectors expressing short hairpin RNAs (shRNAs) against BRCA1 (NM_007299) were obtained from GeneChem Co., Ltd (Shanghai, China), the synthesized sequences are shown in Supplementary Table S3. The nonsilencing shRNA sequence was used as a negative control; the synthesized sequences are shown in Supplementary Table S3. The shRNA lentiviral particles for Nampt (sc45843-V) and SIRT1 (sc-40986-V) were purchased from Santa Cruz Biotechnology (CA, USA). For overexpression of BRCA1, Nampt or SIRT1, the open reading frames of BRCA1 (NM_007299), Nampt (NM_005746) or SIRT1 (NM_012238) were respectively cloned into the lentiviral vector GV287 (Ubi-MCS-3FLAG-SV40-EGFP; GeneChem Co., Ltd). Transfections were performed using polybrene and enhanced infection solution (GeneChem Co., Ltd) according to the manufacturer's recommended protocol. The efficiency of BRCA1, Nampt or SIRT1 knockdown and overexpression and the procedures were as previously reported ${ }^{17}$.

Real-time quantitative PCR. Total RNA was extracted using Trizol reagents (Invitrogen) according to the manufacturer's protocol. DNA contamination was removed by adding DNase I (Invitrogen) according to the manufacturer's protocol. Total RNA was then reverse-transcribed from $2 \mu \mathrm{g}$ of RNA using the PrimeScript RT Master Mix kit (TaKaRa, Dalian, China) and amplified by SYBR Premix Ex TaqTM II (TaKaRa) in a Roche LightCycler 2.0 instrument (Roche Diagnostics, Mannheim, Germany). The specific primer sequences for BRCA1, SIRT1 and Nampt were shown in Supplementary Table S3. GAPDH mRNA was amplified as an internal control for normalization of each sample. All samples were analyzed in triplicate using the $2^{-\Delta \Delta \mathrm{CT}}$ method. 
(a)
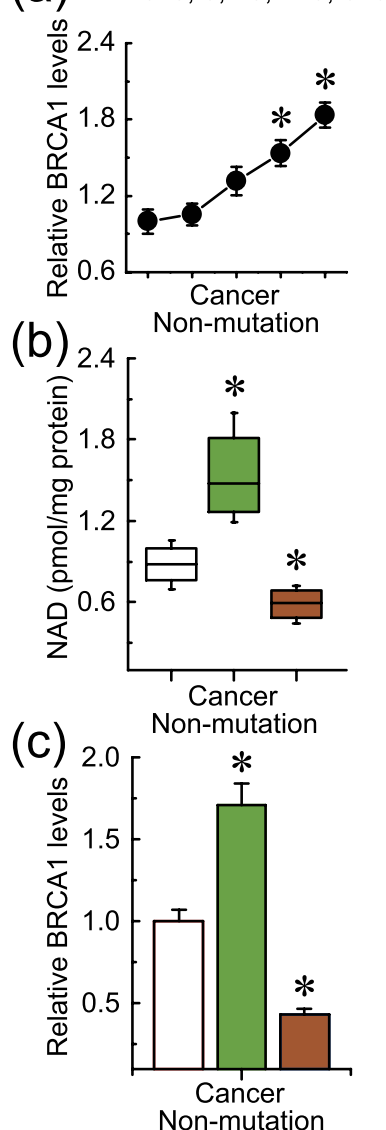
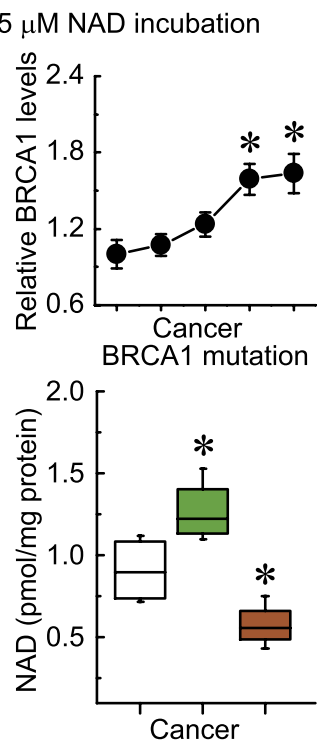

BRCA1 mutation

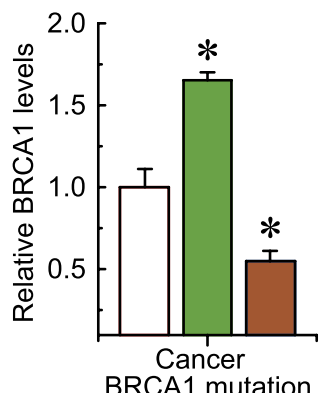

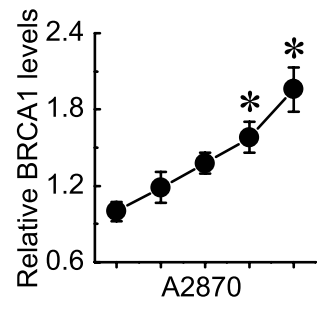
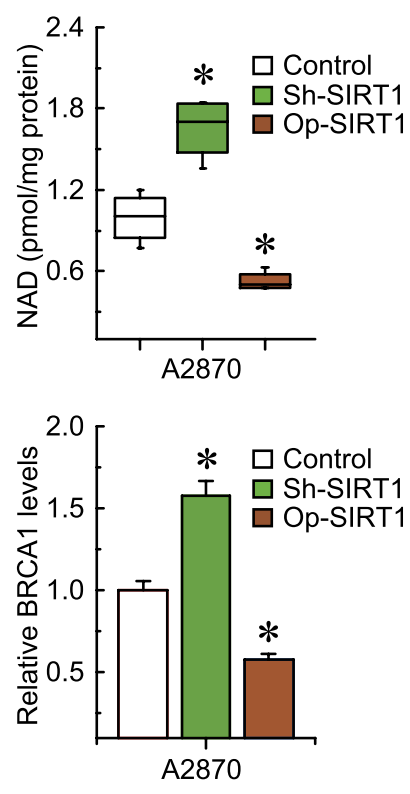

Figure 5 Effects of SIRT1-mediated intracellular NAD consumption on BRCA1 levels. (a), BRCA1 levels after incubation with different concentrations of NAD in A2780 cells (repeated 12 times) and primary non-BRCA1-mutated and BRCA1-mutated ovarian cancer cells ( $\mathrm{n}=12$ ). 1-5: incubation with 0, $5,25,125$, or $625 \mu \mathrm{M}$ NAD. Bar graphs show mean \pm SD. ${ }^{*} P<0.05 v$ s. control. (b and c), NAD and BRCA1 levels after knockdown or overexpression of SIRT1 in A2780 cells (repeated 12 times) and primary non-BRCA1-mutated and BRCA1-mutated ovarian cancer cells $(\mathrm{n}=12)$. Bar graphs show mean \pm SD. Sh, shRNAs; Op, overexpression. ${ }^{*} P<0.05$ vs. control.

Western blotting for SIRT1. Briefly, $50 \mu \mathrm{g}$ protein was separated by $8 \%$ SDS polyacrylamide gels, and transferred to polyvinyl difluoride membranes (Millipore Corp.). The membranes were blocked in TBS containing $0.1 \%$ Tween- 20 and $5 \%$ nonfat dry milk for $30 \mathrm{~min}$ at room temperature, and incubated with antibody to SIRT1 (sc-15404) (1:200; Santa Cruz Biotechnology) overnight at $4{ }^{\circ} \mathrm{C}$. Then, the membranes were washed by PBS-Tween followed by $1 \mathrm{~h}$ incubation at room temperature with horseradish peroxidase-conjugated secondary antibody $(1: 5000$ Santa Cruz Biotechnology) and detected using the enhanced chemiluminescence (Amersham Life Science).
Bisulfite sequencing for BRCA1 promoter. All the tissues were used for bisulfite sequencing from the non-BRCA1-mutated cases. Genomic DNA extracted from ovarian cancer and normal ovarian tissue with a TIANamp Genomic DNA kit (Tiangen Biotech, Beijing, China) was subjected to bisulfite conversion using the EZ DNA Methylation-Direct kit (Zymo Research, Orange, USA) following the manufacturer's instructions; the conversion efficiency was estimated to be at least 99.6\%. It was then amplified by nested PCR. After gel purification, cloning and transformation into E. coli Competent Cells JM109 (TaKaRa), ten positive clones of each sample were sequenced to ascertain the methylation patterns of each $\mathrm{CpG}$ locus.

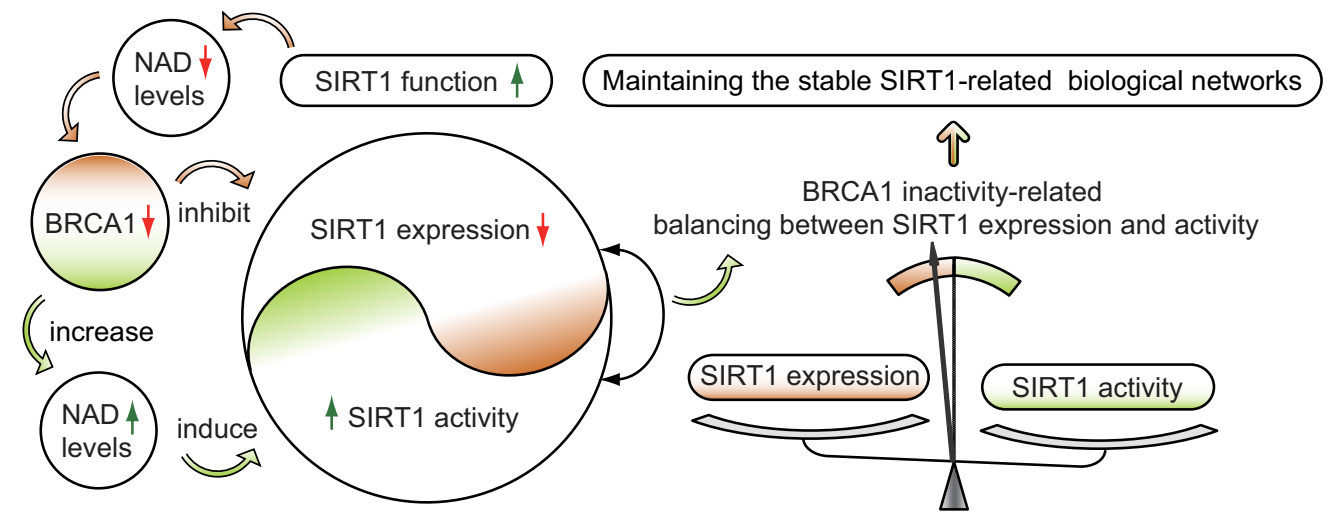

Figure 6 Proposed model of crosstalk between BRCA1 and SIRT1 in ovarian cancer. BRCA1 inactivation events (mutation, promoter methylation, or other pathways) will inhibit SIRT1 expression. However, BRCA1 inactivation induces a substantial increase in NAD levels, and consequently enhances SIRT1 activity. This is a special compensatory mechanism for the loss of SIRT1 expression. The model shows a significant effect of BRCA1 in the maintenance of SIRT1-related biological processes in ovarian cancer. The changes are indicated by red and green arrows. 
The primers were used for BRCA1 gene promoter (Accession number: NG_005905) were shown in Supplementary Table S3. The conditions were as follows: $95^{\circ} \mathrm{C}$ for 2 min, 40 cycles of $30 \mathrm{~s}$ at $95^{\circ} \mathrm{C}, 30 \mathrm{~s}$ at $56^{\circ} \mathrm{C}$ and $45 \mathrm{~s}$ at $72^{\circ} \mathrm{C}$, then $72^{\circ} \mathrm{C}$ for $7 \mathrm{~min}$.

NAD incubation. A2780 and primary ovarian cancer cells were incubated with 0,5 , 25,125 or $625 \mu \mathrm{M}$ NAD (Sigma, CA, USA) for $3 \mathrm{~h}$ at $37^{\circ} \mathrm{C}$.

NAD levels assay. For NAD assay, $20 \mathrm{mg}$ of freezed ovarian tissue, or $20 \mu \mathrm{l}$ packed cultured cells were homogenized in $400 \mu \mathrm{l}$ BioVsion NAD/NADH Extraction Buffer (BioVsion, CA, USA). The homogenate was ultrafiltered using BioVsion 10-kD cutoff filters $\left(14000 \mathrm{~g}, 30 \mathrm{~min}, 4^{\circ} \mathrm{C}\right)$. Assays were performed using the NAD/NADH Quantification Kits according to the manufacturer's instructions (BioVsion)

SIRT1 activity assay. SIRT1 deacetylase activity was evaluated by using a commercially available CycLex SIRT1/Sir2 Deacetylase Fluorometric Assay Kit (Cyclex Co., Ltd., Nagano, Japan), according to the manufacturer's protocols.

Statistical analysis. Regression analysis was used to examine the possible relationship between BRCA1 levels or NAD levels and SIRT1 levels or SIRT1 activity in ovarian cancer samples. The data are presented as means \pm standard deviations (SD). Statistical differences in the data were evaluated by Student's t-test or one-way analysis of variance as appropriate, and were considered significant at $P<0.05$.

1. Lech, A. et al. Ovarian cancer as a genetic disease. Front. Biosci. 18, 543-563 (2013)

2. Pruthi, S., Gostout, B. S. \& Lindor, N. M. Identification and Management of Women With BRCA Mutations or Hereditary Predisposition for Breast and Ovarian Cancer. Mayo Clin. Proc. 85, 1111-1120 (2010).

3. Dacheux, E. et al. BRCA1-Dependent Translational Regulation in Breast Cancer Cells. PLoS One 8, e67313 (2013).

4. Bi, F. F., Li, D. \& Yang, Q. Promoter hypomethylation, especially around the E26 transformation-specific motif, and increased expression of poly (ADP-ribose) polymerase 1 in BRCA-mutated serous ovarian cancer. BMC Cancer 13, 90 (2013)

5. Fang, Y. Y., Li, D., Cao, C., Li, C. Y. \& Li, T. T. Glucocorticoid receptor repression mediated by BRCA1 inactivation in ovarian cancer. BMC Cancer 14, 188 (2014).

6. Li, D. et al. Poly (ADP-ribose) polymerase 1 transcriptional regulation: a novel crosstalk between histone modification $\mathrm{H} 3 \mathrm{~K} 9 \mathrm{ac}$ and ETS1 motif hypomethylation in BRCA1-mutated ovarian cancer. Oncotarget 5, 291-297 (2014).

7. Li, D. et al. Effect of BRCA1 on epidermal growth factor receptor in ovarian cancer. J. Exp. Clin. Cancer Res. 32, 102 (2013).

8. Li, D. et al. Regulation of DNA methyltransferase 1 transcription in BRCA1mutated breast cancer: a novel crosstalk between E2F1 motif hypermethylation and loss of histone H3 lysine 9 acetylation. Mol. Cancer 13, 26 (2014).

9. Li, D. et al. Epigenetic repression of phosphatidylethanolamine, Nmethyltransferase (PEMT) in BRCA1-mutated breast cancer. Oncotarget 5 , 1315-25 (2014).

10. Revollo, J. R. \& Li, X. The ways and means that fine tune Sirt1 activity. Trends Biochem. Sci. 38, 160-167 (2013).

11. Sun, L. et al. PIASy mediates hypoxia-induced SIRT1 transcriptional repression and epithelial-to-mesenchymal transition in ovarian cancer cells. J. Cell Sci. 126, 3939-47 (2003).

12. Menendez, J. A., Mehmi, I., Atlas, E., Colomer, R. \& Lupu, R. Novel signaling molecules implicated in tumor-associated fatty acid synthase-dependent breast cancer cell proliferation and survival: Role of exogenous dietary fatty acids, p53p21WAF1/CIP1, ERK1/2 MAPK, p27KIP1, BRCA1, and NF-kappaB. Int. J. Oncol. 24, 591-608 (2004).

13. Tang, M. K. et al. BRCA1 deficiency induces protective autophagy to mitigate stress and provides a mechanism for BRCA1 haploinsufficiency in tumorigenesis. Cancer Lett. 346, 139-147 (2014).

14. Marks, J. R. Refining the role of BRCA1 in combating oxidative stress. Breast Cancer Res. 15, 320 (2013).

15. Zou, J., Rezvani, K., Wang, H., Lee, K. S. \& Zhang, D. BRCA1 downregulates the kinase activity of Polo-like kinase 1 in response to replication stress. Cell Cycle 12, 2255-2265 (2013).
16. Chang, H. C. \& Guarente, L. SIRT1 and other sirtuins in metabolism. Trends Endocrinol. Metab. 25, 138-145 (2014).

17. Li, D. et al. BRCA1 as a nicotinamide adenine dinucleotide (NAD)-dependent metabolic switch in ovarian cancer. Cell Cycle 13, 2564-2571 (2014).

18. Martinez-Outschoorn, U. E. et al. Hereditary ovarian cancer and twocompartment tumor metabolism: epithelial loss of BRCA1 induces hydrogen peroxide production, driving oxidative stress and NFkappaB activation in the tumor stroma. Cell Cycle 11, 4152-4166 (2012).

19. Ou, X., Lee, M. R., Huang, X., Messina-Graham, S. \& Broxmeyer, H. E. SIRT1 positively regulates autophagy and mitochondria function in embryonic stem cells under oxidative stress. Stem Cells 32, 1183-1194 (2014).

20. Fan, S., Meng, Q., Auborn, K., Carter, T. \& Rosen, E. M. BRCA1 and BRCA2 as molecular targets for phytochemicals indole-3-carbinol and genistein in breast and prostate cancer cells. Br. J. Cancer 94, 407-426 (2006).

21. Jung, T. W., Lee, K. T., Lee, M. W. \& Ka, K. H. SIRT1 attenuates palmitate-induced endoplasmic reticulum stress and insulin resistance in HepG2 cells via induction of oxygen-regulated protein 150. Biochem. Biophys. Res. Commun. 422, 229-232 (2012).

22. Lee, J. et al. Exendin-4 attenuates endoplasmic reticulum stress through a SIRT1dependent mechanism. Cell Stress Chaperones 19, 649-656 (2014).

23. Chiarugi, A., Dolle, C., Felici, R. \& Ziegler, M. The NAD metabolome-a key determinant of cancer cell biology. Nat. Rev. Cancer 12, 741-752 (2012).

24. Han, C. et al. Role of mammalian sirtuin 1 (SIRT1) in lipids metabolism and cell proliferation of goose primary hepatocytes. Mol. Cell Endocrinol. 382, 282-291 (2014).

25. Burga, L. N. et al. Altered proliferation and differentiation properties of primary mammary epithelial cells from BRCA1 mutation carriers. Cancer Res. 69 , 1273-1278 (2009).

26. Promkan, M., Liu, G., Patmasiriwat, P. \& Chakrabarty, S. BRCA1 modulates malignant cell behavior, the expression of survivin and chemosensitivity in human breast cancer cells. Int. J. Cancer 125, 2820-2828 (2009).

27. Hiraike, H. et al. Identification of DBC1 as a transcriptional repressor for BRCA1. Br. J. Cancer 102, 1061-1067 (2010).

28. Wang, R. H. et al. Interplay among BRCA1, SIRT1, and Survivin during BRCA1associated tumorigenesis. Mol. Cell 32, 11-20 (2008).

\section{Acknowledgments}

This work was supported by the Natural Science Foundation of China (No. 81402130) and Doctoral Start-up Foundation of Liaoning Province (No. 20141045).

\section{Author contribution}

D.L. and Q.Y. designed the study. D.L., F.F.B., N.N.C., J.M.C., W.P.S., Y.M.Z. and C.Y.L. carried out data acquisition and interpretation. D.L. and F.F.B. wrote the paper. All authors reviewed the manuscript

\section{Additional information}

Supplementary information accompanies this paper at http://www.nature.com/ scientificreports

Competing financial interests: The authors declare no competing financial interests. How to cite this article: Li, D. et al. A novel crosstalk between BRCA1 and sirtuin 1 in ovarian cancer. Sci. Rep. 4, 6666; DOI:10.1038/srep06666 (2014)

cc)(1) This work is licensed under a Creative Commons Attribution-NonCommercialShareAlike 4.0 International License. The images or other third party material in this article are included in the article's Creative Commons license, unless indicated otherwise in the credit line; if the material is not included under the Creative Commons license, users will need to obtain permission from the license holder in order to reproduce the material. To view a copy of this license, visit http:// creativecommons.org/licenses/by-nc-sa/4.0/ 